University of Wollongong

Research Online

Faculty of Engineering and Information

Faculty of Engineering and Information

Sciences - Papers: Part A

Sciences

$1-1-2016$

Internal energy transfer in dynamical behavior of slightly curved shear deformable microplates

Mergen H. Ghayesh

University of Wollongong, mergen@uow.edu.au

Hamed Farokhi

McGill University

Gursel Alici

University of Wollongong, gursel@uow.edu.au

Follow this and additional works at: https://ro.uow.edu.au/eispapers

Part of the Engineering Commons, and the Science and Technology Studies Commons

Research Online is the open access institutional repository for the University of Wollongong. For further information contact the UOW Library: research-pubs@uow.edu.au 


\title{
Internal energy transfer in dynamical behavior of slightly curved shear deformable microplates
}

\begin{abstract}
This paper investigates the internal energy transfer and modal interactions in the dynamical behavior of slightly curved microplates. Employing the third-order shear deformation theory, the microplate model is developed taking into account geometric nonlinearities as well as the modified couple stress theory; the initial curvature is modeled by an initial imperfection in the out-of-plane direction. The in-plane displacements and inertia are retained, and the coupled out-of-plane, rotational, and in-plane motion characteristics are analyzed. Specifically, continuous models are developed for kinetic and potential energies as well as damping and external works; these are balanced and reduced via Lagrange's equations along with an assumed-mode technique. The reduced-order model is then solved numerically by means of a continuation technique; stability analysis is performed by means of the Floquet theory. The possibility of the occurrence of modal interactions and internal energy transfers is verified via a linear analysis on different natural frequencies of the system. The nonlinear resonant response of the system is obtained for the cases with internal energy transfer, and energy transfer mechanisms are analyzed; as we shall see, the presence of an initial curvature affects the system dynamics substantially. The importance of taking into account small-size effects is also shown by discovering this fact that both the linear and nonlinear internal energy transfer mechanisms are shifted substantially if this effect is ignored.
\end{abstract}

\section{Keywords}

curved, slightly, microplates, behavior, deformable, dynamical, transfer, energy, internal, shear

\section{Disciplines}

Engineering | Science and Technology Studies

\section{Publication Details}

Ghayesh, M. H., Farokhi, H. \& Alici, G. (2016). Internal energy transfer in dynamical behavior of slightly curved shear deformable microplates. Journal of Computational and Nonlinear Dynamics, 11 (4), 041002-1-041002-11. 


\author{
Mergen H. Ghayesh ${ }^{1}$ \\ School of Mechanical, Materials, and \\ Mechatronic Engineering, \\ University of Wollongong, \\ Wollongong, NSW 2522, Australia \\ e-mail: mergen@uow.edu.au \\ Hamed Farokhi \\ Department of Mechanical Engineering, \\ McGill University, \\ Montreal, QC H3A OC3, Canada \\ e-mail: hamed.farokhi@mail.mcgill.ca

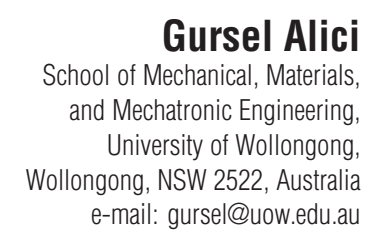

This paper investigates the internal energy transfer and modal interactions in the dynamical behavior of slightly curved microplates. Employing the third-order shear deformation theory, the microplate model is developed taking into account geometric nonlinearities as well as the modified couple stress theory; the initial curvature is modeled by an initial imperfection in the out-of-plane direction. The in-plane displacements and inertia are retained, and the coupled out-of-plane, rotational, and in-plane motion characteristics are analyzed. Specifically, continuous models are developed for kinetic and potential energies as well as damping and external works; these are balanced and reduced via Lagrange's equations along with an assumed-mode technique. The reduced-order model is then solved numerically by means of a continuation technique; stability analysis is performed by means of the Floquet theory. The possibility of the occurrence of modal interactions and internal energy transfers is verified via a linear analysis on different natural frequencies of the system. The nonlinear resonant response of the system is obtained for the cases with internal energy transfer, and energy transfer mechanisms are analyzed; as we shall see, the presence of an initial curvature affects the system dynamics substantially. The importance of taking into account small-size effects is also shown by discovering this fact that both the linear and nonlinear internal energy transfer mechanisms are shifted substantially if this effect is ignored. [DOI: 10.1115/1.4031290]

Keywords: internal energy transfer, size-dependent dynamical behavior, shear deformable microplate, stability

\section{Introduction}

Due to new technological advances in microfabrication, microscale continuous elements such as microbeams and microplates are widely used in many advanced micromachine components and engineering devices [1-18]. Microplates are present, for example, in biosensors, biomechanical organs, microactuators, and microswitches. These widespread applications have motivated a substantial amount of research on the dynamical behavior of this class of microscale continuous elements.

Experimental investigations [19-21] showed that microscale continuous elements display size-dependent deformation behavior, which cannot be predicted by classical continuum theories. This necessitated the development of new continuum theories such as the strain gradient [22-25] and modified couple stress [26-28] theories.

In general, due to improper manufacturing process, it is very likely to make slightly curved microplates, even if it is not intended to do so. Moreover, in some applications, such as in microswitches, microshutters, and bandpass filters, the microplate is manufactured in a curved shape. This geometric imperfection is taken into account in this analysis by an initial curvature in the out-of-plane direction; it is explored that how this initial curvature changes the dynamical behavior of the microplate.

The literature regarding the dynamical behavior of initially perfectly flat microplates is quite large, for the cases where linear systems are considered; however, it is not large where nonlinear systems are considered. Reviewing some of the recent papers

\footnotetext{
${ }^{1}$ Corresponding author.

Contributed by the Design Engineering Division of ASME for publication in the Journal of Computational and Nonlinear Dynamics. Manuscript received February 25, 2015; final manuscript received August 5, 2015; published online November 13, 2015. Assoc. Editor: Ahmet S. Yigit.
}

which analyzed the dynamical behavior of perfectly flat microplates, starting with the linear analyses, Hashemi and Samaei [29] investigated the linear buckling of microplates subject to an inplane excitation load, based on the nonlocal Mindlin plate theory. Sharma and Sharma [30] analyzed the transverse linear dynamics of a generalized thermoelastic circular plate subject to different temperature. He et al. [31] developed a size-dependent fourvariable functionally graded microplates employing the modified couple stress theory and Hamilton's principle; they obtained the closed form solutions for linear bending, buckling, and free dynamics of a simply supported microplate. Wang et al. [32] derived a size-dependent microplate model by means of the Kirchhoff plate theory based on a strain gradient elasticity theory. Ramezani [33] employed a first-order shear deformation theory in order to model the motion characteristics of a microplate via use of a strain gradient elasticity theory. Tahani et al. [34] employed the modified couple stress theory in order to investigate the size-dependent free dynamic characteristics of rectangular microplates predeformed by an electric field. Roque et al. [35] examined the bending of a shear deformable microplate on the basis of the modified couple stress theory. The application of nonlinear theories to the dynamical behavior of microplates led to few published papers in the literature; for instance, Asghari [36] obtained the sizedependent equations of motion of microplates on the basis of the modified couple stress theory. Thai and Choi [37] developed a size-dependent model of functionally graded Kirchhoff Mindlin plates via use of the modified couple stress theory. In both of the valuable studies in Refs. [36,37], only the equations of motion were derived, and no solutions for the nonlinear dynamics were obtained. Ansari et al. [38] examined the nonlinear bending and postbuckling of functionally graded Mindlin rectangular plates based on the modified couple stress theory. There have been several studies on the dynamical behavior of initially curved 
microbeams (MEMS microarches), for example, by Das and Batra [39], who examined the pull-in and snap-through instabilities in MEMS shallow arches under dynamic loading; Ouakad and Younis [40,41] and Ouakad [42], who analyzed the dynamic behavior of an arch resonator and its application as a band-pass filter; Mohammad and Ouakad [43], who performed a bifurcation analysis of electrostatically actuated MEMS arches; and Medina et al. [44-46], who performed theoretical and experimental investigations on the static behavior of electrostatically actuated initially curved microbeams. However, the literature regarding the dynamical behavior of initially curved microplates is limited [15].

An important issue which has never been studied in the dynamical behavior of microplates is the internal energy transfer and internal resonances. This paper examines the internal energy transfer and modal interactions in the dynamical behavior of slightly curved shear deformable microplates, with special consideration to the effect of the initial curvature on the internal energy transfer mechanisms, and employing the third-order shear deformation theory. Taking into account small-size effects, via use of the modified couple stress theory, the elastic potential energy of the continuous system is obtained; the kinetic energy as well as the work due to the damping and external excitation forces is also obtained as continuous functions of the displacement field. The contribution of the initial curvature, modeled as a slight initial displacement in the out-of-plane direction, in the potential energy is included in the modeling. The Lagrange equations along with an assumed-mode technique are employed to obtain a reduced-order model. A linear analysis is performed to verify the possibility of the occurrence of internal energy transfer and modal interactions. The pseudo-arclength continuation technique is then employed to solve the reduced-order model of the system; stability analysis is conducted using the Floquet theory. For the systems tuned to internal energy transfer, the results are illustrated in the form of frequency-responses and force-responses. As we shall see, an interesting nonlinear dynamical behavior, with multiple solution branches due to internal energy transfer mechanisms, is displayed by the system. Differences and similarities between the steadystate coupled dynamical response of the initially perfectly flat and initially curved microplates are highlighted via comparing the corresponding frequency-response and force-response curves; this illustrates that how even small imperfections may change the system dynamics and the internal energy transfer substantially.

\section{Problem Statement and Displacement Field}

Schematic representation of the system is shown in Fig. 1; the system consists of a microplate with in-plane dimensions $a$ and $b$ in the $x$ and $y$ directions, respectively, thickness $h$, mass density $\rho$, Poisson's ratio $\nu$, length-scale parameter $l$, and Young's modulus $E$. A Cartesian coordinate system $(O ; x, y, z)$, with the origin $O$ at one corner, defines the displacement field; $x$ and $y$ axes define the midplane coordinates while $z$-axis defines the out-of-plane coordinate from the midplane, from the static equilibrium state. The displacement components of each point of the midplane of the microplate in the $x, y$, and $z$ directions are denoted by

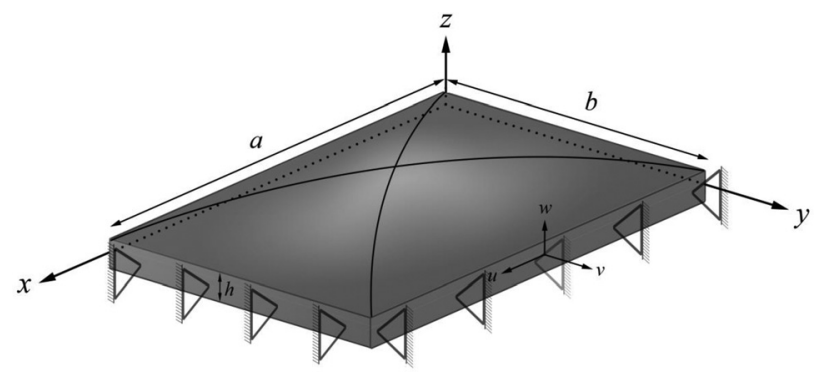

Fig. 1 Schematic representation of an initially slightly curved shear deformable microplate $u=u(x, y, t), v=v(x, y, t)$, and $w=w(x, y, t)$, respectively, $t$ is time. $\phi_{1}=\phi_{1}(x, y, t)$ and $\phi_{2}=\phi_{2}(x, y, t)$ also denote the rotations of the transverse normal at $z=0$ about the $y$ and $x$ axes, respectively. A distributed harmonically varying force per unit area, $f_{1} \cos (\omega t)$, is exerted to the microplate in the $z$ direction, where $f_{1}$ and $\omega$ are in $\mathrm{N} / \mathrm{m}^{2}$ and $\mathrm{rad} / \mathrm{s}$, respectively. An initial curvature in the positive out-of-plane direction in the form of $w_{0}(x, y)=A_{0} \sin (\pi x / a) \sin (\pi y / b)$ is considered. The components of the displacement vector (u) of a generic point (in the $x, y$, and $z$ directions, respectively) of a geometrically imperfect third-order shear deformable microplate are given by

$$
\begin{gathered}
u_{1}(x, y, z, t)=u+z \phi_{1}-\frac{4}{3 h^{2}} z^{3}\left(\phi_{1}+\frac{\partial w}{\partial x}\right) \\
u_{2}(x, y, z, t)=v+z \phi_{2}-\frac{4}{3 h^{2}} z^{3}\left(\phi_{2}+\frac{\partial w}{\partial y}\right) \\
u_{3}(x, y, z, t)=w+w_{0}
\end{gathered}
$$

\section{Expressions for Kinetic Energy, External Work, and Damping}

The kinetic energy of a geometrically imperfect shear deformable microplate can be modeled as

$$
\begin{aligned}
T= & \frac{1}{2} \rho h \int_{0}^{a} \int_{0}^{b}\left\{\left(\frac{\partial u}{\partial t}\right)^{2}+\left(\frac{\partial v}{\partial t}\right)^{2}+\left(\frac{\partial w}{\partial t}\right)^{2}+\frac{17 h^{2}}{315}\right. \\
& \times\left[\left(\frac{\partial \phi_{1}}{\partial t}\right)^{2}+\left(\frac{\partial \phi_{2}}{\partial t}\right)^{2}\right]-\frac{8 h^{2}}{315}\left(\frac{\partial \phi_{1}}{\partial t} \frac{\partial^{2} w}{\partial x \partial t}+\frac{\partial \phi_{2}}{\partial t} \frac{\partial^{2} w}{\partial y \partial t}\right) \\
& \left.+\frac{h^{2}}{252}\left[\left(\frac{\partial^{2} w}{\partial x \partial t}\right)^{2}+\left(\frac{\partial^{2} w}{\partial y \partial t}\right)^{2}\right]\right\} \mathrm{d} x \mathrm{~d} y
\end{aligned}
$$

The work of the external distributed harmonic excitation load per unit area, $f_{1} \cos (\omega t)$, acting on the system in the positive $z$ direction, can be formulated as

$$
W=\int_{0}^{a} \int_{0}^{b}\left(f_{1} \cos (\omega t) w\right) \mathrm{d} x \mathrm{~d} y
$$

A nonconservative external viscous damping with the coefficient of $c$ is introduced to the model via using the Rayleigh dissipation function as

$$
\begin{aligned}
F= & \frac{1}{2} c \int_{0}^{a} \int_{0}^{b}\left[\left(\frac{\partial u}{\partial t}\right)^{2}+\left(\frac{\partial v}{\partial t}\right)^{2}+\left(\frac{\partial w}{\partial t}\right)^{2}\right. \\
& \left.+\frac{17}{315} h^{2}\left(\left(\frac{\partial \phi_{1}}{\partial t}\right)^{2}+\left(\frac{\partial \phi_{2}}{\partial t}\right)^{2}\right)\right] \mathrm{d} x \mathrm{~d} y
\end{aligned}
$$

\section{Continuous Model for Elastic Potential Energy Via Modified Couple Stress Theory}

In this section, a continuous model for the elastic strain energy stored in the microplate is proposed based on the modified couple stress theory.

The strain energy of the microplate, on the basis of the modified couple stress theory [47], can be formulated as

$$
U=\frac{1}{2} \int_{V}(\boldsymbol{\sigma}: \boldsymbol{\varepsilon}+\mathbf{m}: \chi) \mathrm{d} v
$$

in which $\boldsymbol{\sigma}$ and $\boldsymbol{\varepsilon}$ denote the stress and strain tensors, respectively; $\mathbf{m}$ and $\chi$ denote the deviatoric part of the symmetric couple stress 
and the symmetric curvature tensors, respectively. The expanded form of Eq. (7) is given by

$$
\begin{aligned}
U=\frac{1}{2} \int_{0}^{a} \int_{0}^{b} & \left\{\int _ { - h / 2 } ^ { h / 2 } \left[\left(\sigma_{x x} \varepsilon_{x x}+\sigma_{y y} \varepsilon_{y y}+\tau_{x y} \gamma_{x y}+\tau_{x z} \gamma_{x z}+\tau_{y z} \gamma_{y z}\right)\right.\right. \\
& +\left(m_{x x} \chi_{x x}+m_{y y} \chi_{y y}+m_{z z} \chi_{z z}+2 m_{x y} \chi_{x y}+2 m_{x z} \chi_{x z}\right. \\
& \left.\left.\left.+2 m_{y z} \chi_{y z}\right)\right] \mathrm{~d} z\right\} \mathrm{d} x \mathrm{~d} y
\end{aligned}
$$

The symmetric curvature tensor $\chi$ is related to the rotation vector $\boldsymbol{\theta}$ by

$$
\boldsymbol{\chi}=\frac{1}{2}\left[\begin{array}{ccc}
2 \frac{\partial \theta_{x}}{\partial x} & \left(\frac{\partial \theta_{x}}{\partial y}+\frac{\partial \theta_{y}}{\partial x}\right) & \left(\frac{\partial \theta_{x}}{\partial z}+\frac{\partial \theta_{z}}{\partial x}\right) \\
\left(\frac{\partial \theta_{x}}{\partial y}+\frac{\partial \theta_{y}}{\partial x}\right) & 2 \frac{\partial \theta_{y}}{\partial y} & \left(\frac{\partial \theta_{y}}{\partial z}+\frac{\partial \theta_{z}}{\partial y}\right) \\
\left(\frac{\partial \theta_{x}}{\partial z}+\frac{\partial \theta_{z}}{\partial x}\right) & \left(\frac{\partial \theta_{y}}{\partial z}+\frac{\partial \theta_{z}}{\partial y}\right) & 2 \frac{\partial \theta_{z}}{\partial z}
\end{array}\right]
$$

where the rotation vector $\boldsymbol{\theta}$ is a function of the displacement field $\mathbf{u}$ as

$$
\boldsymbol{\theta}=\frac{1}{2} \nabla \times \mathbf{u}=\left\{\frac{1}{2}\left(\frac{\partial u_{3}}{\partial y}-\frac{\partial u_{2}}{\partial z}\right), \frac{1}{2}\left(\frac{\partial u_{1}}{\partial z}-\frac{\partial u_{3}}{\partial x}\right), \frac{1}{2}\left(\frac{\partial u_{2}}{\partial x}-\frac{\partial u_{1}}{\partial y}\right)\right\}
$$

Inserting Eqs. (1)-(3) into Eq. (10) and the resultant equations into Eq. (9) gives the components of the symmetric curvature tensor as

$$
\begin{aligned}
& \left\{\begin{array}{l}
\chi_{x x} \\
\chi_{y y} \\
\chi_{z z} \\
\chi_{x y}
\end{array}\right\}=\left\{\begin{array}{l}
\chi_{x x, 0} \\
\chi_{y y, 0} \\
\chi_{z z, 0} \\
\chi_{x y, 0}
\end{array}\right\}+z^{2}\left\{\begin{array}{c}
\kappa_{x}^{(2)} \\
\kappa_{y}^{(2)} \\
\kappa_{z}^{(2)} \\
\kappa_{x y}^{(2)}
\end{array}\right\} \\
& \left\{\begin{array}{l}
\chi_{x z} \\
\chi_{y z}
\end{array}\right\}=\left\{\begin{array}{l}
\chi_{x z, 0} \\
\chi_{y z, 0}
\end{array}\right\}+z\left\{\begin{array}{c}
\kappa_{x z}^{(1)} \\
\kappa_{y z}^{(1)}
\end{array}\right\}+z^{3}\left\{\begin{array}{c}
\kappa_{x z}^{(3)} \\
\kappa_{y z}^{(3)}
\end{array}\right\} \\
& \left\{\begin{array}{c}
\chi_{x x, 0} \\
\chi_{y y, 0} \\
\chi_{z z, 0} \\
\chi_{x y, 0}
\end{array}\right\}=\left\{\begin{array}{c}
-\frac{1}{2} \frac{\partial \phi_{2}}{\partial x}+\frac{1}{2} \frac{\partial^{2} w}{\partial x \partial y} \\
\frac{1}{2} \frac{\partial \phi_{1}}{\partial y}-\frac{1}{2} \frac{\partial^{2} w}{\partial x \partial y} \\
\frac{1}{2}\left(\frac{\partial \phi_{2}}{\partial x}-\frac{\partial \phi_{1}}{\partial y}\right) \\
\frac{1}{4}\left(\frac{\partial \phi_{1}}{\partial x}-\frac{\partial^{2} w}{\partial x^{2}}-\frac{\partial \phi_{2}}{\partial y}+\frac{\partial^{2} w}{\partial y^{2}}\right)
\end{array}\right\} \\
& \left\{\begin{array}{c}
\kappa_{x}^{(2)} \\
\kappa_{y}^{(2)} \\
\kappa_{z}^{(2)} \\
\kappa_{x y}^{(2)}
\end{array}\right\}=\left\{\begin{array}{c}
\frac{2}{h^{2}}\left(\frac{\partial \phi_{2}}{\partial x}+\frac{\partial^{2} w}{\partial x \partial y}\right) \\
-\frac{2}{h^{2}}\left(\frac{\partial \phi_{1}}{\partial y}+\frac{\partial^{2} w}{\partial x \partial y}\right) \\
-\frac{2}{h^{2}}\left(\frac{\partial \phi_{2}}{\partial x}-\frac{\partial \phi_{1}}{\partial y}\right) \\
-\frac{1}{h^{2}}\left(\frac{\partial \phi_{1}}{\partial x}+\frac{\partial^{2} w}{\partial x^{2}}-\frac{\partial \phi_{2}}{\partial y}-\frac{\partial^{2} w}{\partial y^{2}}\right)
\end{array}\right\}
\end{aligned}
$$

$$
\begin{gathered}
\left\{\begin{array}{l}
\chi_{x z, 0} \\
\chi_{y z, 0}
\end{array}\right\}=\left\{\begin{array}{l}
\frac{1}{4}\left(\frac{\partial^{2} v}{\partial x^{2}}-\frac{\partial^{2} u}{\partial x \partial y}\right) \\
1 \\
\frac{1}{4}\left(\frac{\partial^{2} v}{\partial x \partial y}-\frac{\partial^{2} u}{\partial y^{2}}\right)
\end{array}\right\} \\
\left\{\begin{array}{c}
\kappa_{x z}^{(1)} \\
\kappa_{y z}^{(1)}
\end{array}\right\}=\left\{\begin{array}{l}
-\frac{1}{4}\left(\frac{\partial^{2} \phi_{1}}{\partial x \partial y}-\frac{\partial^{2} \phi_{2}}{\partial x^{2}}\right)+\frac{2}{h^{2}}\left(\phi_{2}+\frac{\partial w}{\partial y}\right) \\
-\frac{1}{4}\left(\frac{\partial^{2} \phi_{1}}{\partial y^{2}}-\frac{\partial^{2} \phi_{2}}{\partial x \partial y}\right)-\frac{2}{h^{2}}\left(\phi_{1}+\frac{\partial w}{\partial x}\right)
\end{array}\right\} \\
\left\{\begin{array}{l}
\kappa_{x z}^{(3)} \\
\kappa_{y z}^{(3)}
\end{array}\right\}=\left\{\begin{array}{c}
\frac{1}{3 h^{2}}\left(\frac{\partial^{2} \phi_{1}}{\partial x \partial y}-\frac{\partial^{2} \phi_{2}}{\partial x^{2}}\right) \\
\frac{1}{3 h^{2}}\left(\frac{\partial^{2} \phi_{1}}{\partial y^{2}}-\frac{\partial^{2} \phi_{2}}{\partial x \partial y}\right)
\end{array}\right\}
\end{gathered}
$$

The strain-displacement relations for the third-order shear deformable microplate with the initial curvature can be expressed as

$$
\begin{aligned}
& \left\{\begin{array}{c}
\varepsilon_{x x} \\
\varepsilon_{y y} \\
\gamma_{x y}
\end{array}\right\}=\left\{\begin{array}{c}
\varepsilon_{x x, 0} \\
\varepsilon_{y y, 0} \\
\gamma_{x y, 0}
\end{array}\right\}+z\left\{\begin{array}{c}
k_{x}^{(1)} \\
k_{y}^{(1)} \\
k_{x y}^{(1)}
\end{array}\right\}+z^{3}\left\{\begin{array}{c}
k_{x}^{(3)} \\
k_{y}^{(3)} \\
k_{x y}^{(3)}
\end{array}\right\} \\
& \left\{\begin{array}{l}
\gamma_{x z} \\
\gamma_{y z}
\end{array}\right\}=\left\{\begin{array}{l}
\gamma_{x z, 0} \\
\gamma_{y z, 0}
\end{array}\right\}+z^{2}\left\{\begin{array}{l}
k_{x z}^{(2)} \\
k_{y z}^{(2)}
\end{array}\right\} \\
& \left\{\begin{array}{c}
\varepsilon_{x x, 0} \\
\varepsilon_{y y, 0} \\
\gamma_{x y, 0}
\end{array}\right\}=\left\{\begin{array}{c}
\frac{\partial u}{\partial x}+\frac{1}{2}\left(\frac{\partial w}{\partial x}\right)^{2}+\frac{\partial w}{\partial x} \frac{\partial w_{0}}{\partial x} \\
\frac{\partial v}{\partial y}+\frac{1}{2}\left(\frac{\partial w}{\partial y}\right)^{2}+\frac{\partial w}{\partial y} \frac{\partial w_{0}}{\partial y} \\
\frac{\partial u}{\partial y}+\frac{\partial v}{\partial x}+\frac{\partial w}{\partial x} \frac{\partial w}{\partial y}+\frac{\partial w}{\partial x} \frac{\partial w_{0}}{\partial y}+\frac{\partial w_{0}}{\partial x} \frac{\partial w}{\partial y}
\end{array}\right\} \\
& \left\{\begin{array}{c}
k_{x}^{(1)} \\
k_{y}^{(1)} \\
k_{x y}^{(1)}
\end{array}\right\}=\left\{\begin{array}{c}
\frac{\partial \phi_{1}}{\partial x} \\
\frac{\partial \phi_{2}}{\partial y} \\
\frac{\partial \phi_{1}}{\partial y}+\frac{\partial \phi_{2}}{\partial x}
\end{array}\right\} \\
& \left\{\begin{array}{l}
k_{x}^{(3)} \\
k_{y}^{(3)} \\
k_{x y}^{(3)}
\end{array}\right\}=\left\{\begin{array}{c}
-\frac{4}{3 h^{2}}\left(\frac{\partial \phi_{1}}{\partial x}+\frac{\partial^{2} w}{\partial x^{2}}\right) \\
-\frac{4}{3 h^{2}}\left(\frac{\partial \phi_{2}}{\partial y}+\frac{\partial^{2} w}{\partial y^{2}}\right) \\
-\frac{4}{3 h^{2}}\left(\frac{\partial \phi_{1}}{\partial y}+\frac{\partial \phi_{2}}{\partial x}+2 \frac{\partial^{2} w}{\partial x \partial y}\right)
\end{array}\right\} \\
& \left\{\begin{array}{l}
\gamma_{x z, 0} \\
\gamma_{y z, 0}
\end{array}\right\}=\left\{\begin{array}{c}
\phi_{1}+\frac{\partial w}{\partial x} \\
\phi_{2}+\frac{\partial w}{\partial y}
\end{array}\right\}
\end{aligned}
$$




$$
\left\{\begin{array}{l}
k_{x z}^{(2)} \\
k_{y z}^{(2)}
\end{array}\right\}=\left\{\begin{array}{c}
-\frac{4}{h^{2}}\left(\phi_{1}+\frac{\partial w}{\partial x}\right) \\
-\frac{4}{h^{2}}\left(\phi_{2}+\frac{\partial w}{\partial y}\right)
\end{array}\right\}
$$

The relation between the deviatoric part of the symmetric couple stress tensor and the symmetric curvature tensor is given by

$$
m_{i j}=\frac{E l^{2}}{1+\nu} \chi_{i j}
$$

The stress-strain relations for an isotropic material, under planestress condition, are given by

$$
\left\{\begin{array}{c}
\sigma_{x x} \\
\sigma_{y y} \\
\tau_{x y} \\
\tau_{x z} \\
\tau_{y z}
\end{array}\right\}=\frac{E}{1-\nu^{2}}\left[\begin{array}{ccccc}
1 & \nu & 0 & 0 & 0 \\
\nu & 1 & 0 & 0 & 0 \\
0 & 0 & (1-\nu) / 2 & 0 & 0 \\
0 & 0 & 0 & (1-\nu) / 2 & 0 \\
0 & 0 & 0 & 0 & (1-\nu) / 2
\end{array}\right]\left\{\begin{array}{l}
\varepsilon_{x x} \\
\varepsilon_{y y} \\
\gamma_{x y} \\
\gamma_{x z} \\
\gamma_{y z}
\end{array}\right\}
$$

The expression for the elastic strain energy of the shear deformable microplate is obtained by inserting Eqs. (11)-(26) into Eq. (8), as follows:

$$
\begin{aligned}
U= & \frac{1}{2} \frac{E h}{1-\nu^{2}} \int_{0}^{a} \int_{0}^{b}\left[\varepsilon_{x x, 0}^{2}+\varepsilon_{y y, 0}^{2}+2 \nu \varepsilon_{x x, 0} \varepsilon_{y y, 0}+\frac{1-\nu}{2}\left(\gamma_{x y, 0}^{2}+\gamma_{x z, 0}^{2}+\gamma_{y z, 0}^{2}\right)\right] \mathrm{d} x \mathrm{~d} y \\
& +\frac{1}{2} \frac{E h^{3}}{12\left(1-\nu^{2}\right)} \int_{0}^{a} \int_{0}^{b}\left[\left(k_{x}^{(1)}\right)^{2}+\left(k_{y}^{(1)}\right)^{2}+2 \nu k_{x}^{(1)} k_{y}^{(1)}+\frac{1-\nu}{2}\left(\left(k_{x y}^{(1)}\right)^{2}+2 \gamma_{x z, 0} k_{x z}^{(2)}+2 \gamma_{y z, 0} k_{y z}^{(2)}\right)\right] \mathrm{d} x \mathrm{~d} y \\
& +\frac{1}{2} \frac{E h^{5}}{80\left(1-\nu^{2}\right)} \int_{0}^{a} \int_{0}^{b}\left[2\left(k_{x}^{(1)} k_{x}^{(3)}+k_{y}^{(1)} k_{y}^{(3)}+\nu k_{x}^{(1)} k_{y}^{(3)}+\nu k_{x}^{(3)} k_{y}^{(1)}\right)+\frac{1-\nu}{2}\left(\left(k_{x z}^{(2)}\right)^{2}+\left(k_{y z}^{(2)}\right)^{2}+2 k_{x y}^{(1)} k_{x y}^{(3)}\right)\right] \mathrm{d} x \mathrm{~d} y \\
& +\frac{1}{2} \frac{E h^{7}}{448\left(1-\nu^{2}\right)} \int_{0}^{a} \int_{0}^{b}\left[\left(k_{x}^{(3)}\right)^{2}+\left(k_{y}^{(3)}\right)^{2}+2 \nu k_{x}^{(3)} k_{y}^{(3)}+\frac{1-\nu}{2}\left(k_{x y}^{(3)}\right)^{2}\right] \mathrm{d} x \mathrm{~d} y \\
& +\frac{1}{2} \frac{E l^{2} h}{1+\nu} \int_{0}^{a} \int_{0}^{b}\left[\chi_{x x, 0}^{2}+\chi_{y y, 0}^{2}+\chi_{z z, 0}^{2}+2 \chi_{x y, 0}^{2}+2 \chi_{x z, 0}^{2}+2 \chi_{y z, 0}^{2}\right] \mathrm{d} x \mathrm{~d} y \\
& +\frac{1}{2} \frac{E l^{2} h^{3}}{6(1+\nu)} \int_{0}^{a} \int_{0}^{b}\left[\chi_{x x, 0} \kappa_{x}^{(2)}+\chi_{y y, 0} \kappa_{y}^{(2)}+\chi_{z z, 0} \kappa_{z}^{(2)}+2 \chi_{x y, 0} \kappa_{x y}^{(2)}+\left(\kappa_{x z}^{(1)}\right)^{2}+\left(\kappa_{y z}^{(1)}\right)^{2}\right] \mathrm{d} x \mathrm{~d} y \\
& +\frac{1}{2} \frac{E l^{2} h^{5}}{80(1+\nu)} \int_{0}^{a} \int_{0}^{b}\left[\left(\kappa_{x}^{(2)}\right)^{2}+\left(\kappa_{y}^{(2)}\right)^{2}+\left(\kappa_{z}^{(2)}\right)^{2}+2\left(\kappa_{x y}^{(2)}\right)^{2}+4 \kappa_{x z}^{(1)} \kappa_{x z}^{(3)}+4 \kappa_{y z}^{(1)} \kappa_{y z}^{(3)}\right] \mathrm{d} x \mathrm{~d} y \\
& +\frac{1}{2} \frac{E l^{2} h^{7}}{224(1+\nu)} \int_{0}^{a} \int_{0}^{b}\left[\left(\kappa_{x z}^{(3)}\right)^{2}+\left(\kappa_{y z}^{(3)}\right)^{2}\right] \mathrm{d} x \mathrm{~d} y
\end{aligned}
$$

\section{Reduced-Order Model}

The analysis is conducted for a shear deformable simplysupported microplate with immovable edges. The following approximate series expansions are assumed for the in-plane and out-of-plane displacements as well as the rotations:

$$
\begin{aligned}
& u(x, y, t)=\sum_{m=1}^{M} \sum_{n=1}^{N} u_{m, n}(t) \sin (m \pi x / a) \sin (n \pi y / b) \\
& v(x, y, t)=\sum_{m=1}^{\hat{M}} \sum_{n=1}^{\hat{N}} v_{m, n}(t) \sin (m \pi x / a) \sin (n \pi y / b) \\
& w(x, y, t)=\sum_{m=1}^{\tilde{M}} \sum_{n=1}^{\tilde{N}} w_{m, n}(t) \sin (m \pi x / a) \sin (n \pi y / b)
\end{aligned}
$$

$$
\begin{aligned}
& \phi_{1}(x, y, t)=\sum_{m=1}^{\tilde{M}} \sum_{n=1}^{\tilde{N}} \phi_{1_{m, n}}(t) \cos (m \pi x / a) \sin (n \pi y / b) \\
& \phi_{2}(x, y, t)=\sum_{m=1}^{\tilde{M}} \sum_{n=1}^{\tilde{N}} \phi_{2_{m, n}}(t) \sin (m \pi x / a) \cos (n \pi y / b)
\end{aligned}
$$

in which $m$ and $n$ are the numbers of half-waves in the $x$ and $y$ directions, respectively, and $u_{m, n}(t), v_{m, n}(t), w_{m, n}(t), \phi_{1_{m, n}}(t)$, and $\phi_{2_{m, n}}(t)$ denote the generalized coordinates. The series expansion defined in Eqs. (28)-(32) satisfy the following boundary conditions of the system:

$$
\begin{array}{llll}
u=v=w=0, & \phi_{2}=0, & M_{x}=0 & \text { at } x=0,
\end{array}
$$

where $M_{x}$ and $M_{y}$ represent the bending moment resultants per unit length. 
The following notation is introduced for brevity:

$$
\mathbf{q}=\left\{u_{m, n}(t), v_{m, n}(t), w_{m, n}(t), \phi_{1_{m, n}}(t), \phi_{2_{m, n}}(t)\right\}^{\mathrm{T}}
$$

in which the dimension of the vector $\mathbf{q}, N$, is the number of degrees of freedom of the reduced-order model.

The Lagrange equations are given by

$$
\frac{\mathrm{d}}{\mathrm{d} t}\left(\frac{\partial T}{\partial \dot{q}_{j}}\right)-\frac{\partial T}{\partial q_{j}}+\frac{\partial U}{\partial q_{j}}+\frac{\partial F}{\partial \dot{q}_{j}}=\frac{\partial W}{\partial q_{j}} \quad j=1, \ldots, N
$$

Substitution of Eqs. (28)-(32) into Eqs. (4)-(6) and (27), and substitution of the resultant expressions into Eq. (36) results in a set of $N$ second-order nonlinear ordinary differential equations with inertial coupling. These equations are solved numerically via the methods explained in Sec. 6.

\section{Solution Techniques}

Application of the procedure explained in Sec. 5 results in $N$ second-order nonlinear ordinary differential equations with
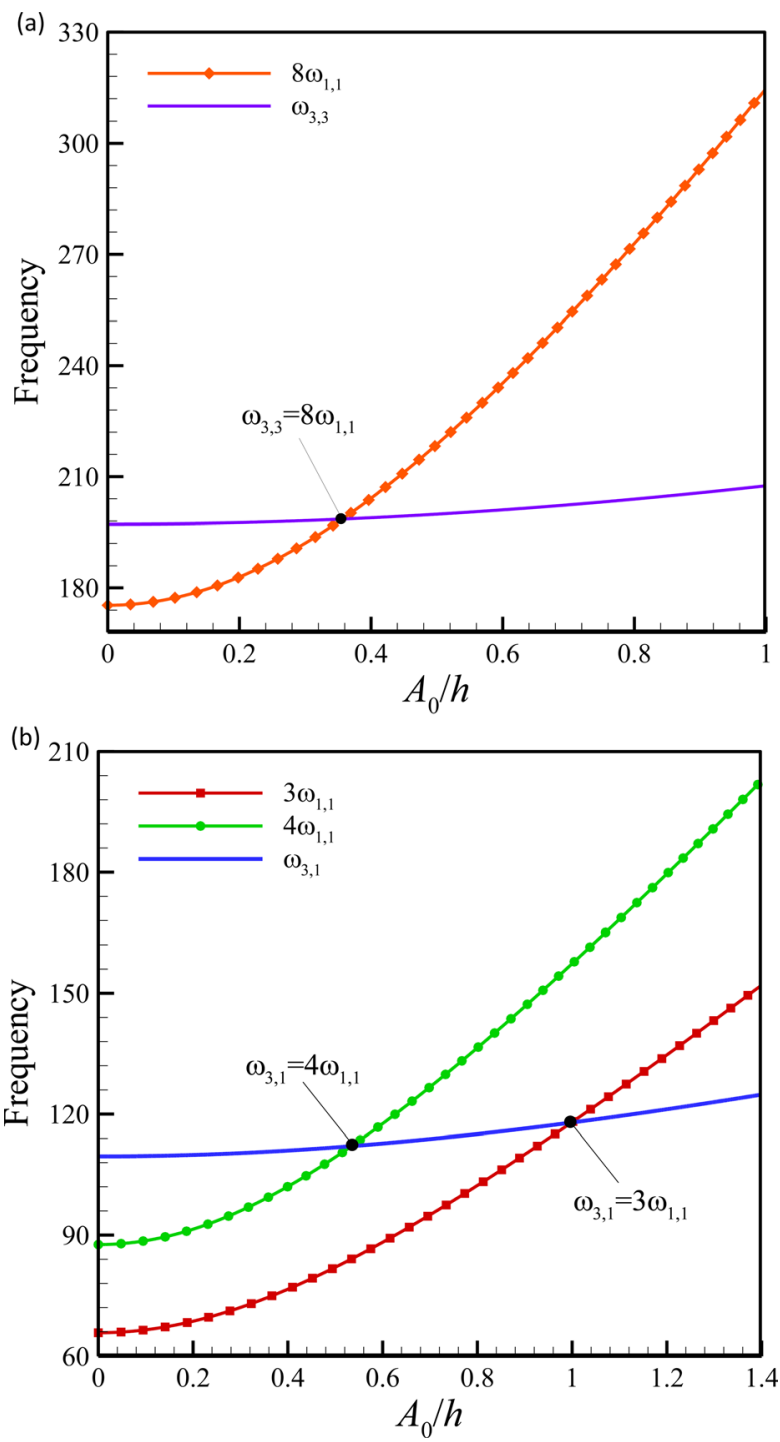

Fig. 2 Variations of the dimensionless natural frequencies of the out-of-plane motion of the microplate with the amplitude of the initial curvature inertial coupling, which can be shown in a matrix equation form with nonlinear coefficients, as follows:

$$
\mathbf{M} \ddot{\mathbf{q}}+\mathbf{C} \dot{\mathbf{q}}+\mathbf{K}_{\mathbf{1}} \mathbf{q}+\mathbf{K}_{\mathbf{2}}(\mathbf{q}) \mathbf{q}+\mathbf{K}_{\mathbf{3}}(\mathbf{q}, \mathbf{q}) \mathbf{q}=\mathbf{f} \cos (\omega t)
$$

where $\mathbf{M}$ is the nondiagonal mass matrix, $\mathbf{C}$ is the damping matrix, and $\mathbf{f}$ is the forcing amplitude vector; $\mathbf{K}_{1}, \mathbf{K}_{2}$, and $\mathbf{K}_{3}$ denote the linear, quadratic, and cubic stiffness matrices, respectively.

Premultiplying both sides of Eq. (37) by $\mathbf{M}^{-1}$ gives the following inertially decoupled matrix equation:

$$
\begin{aligned}
\mathbf{I} \ddot{\mathbf{q}} & +\mathbf{M}^{-1} \mathbf{C} \dot{\mathbf{q}}+\mathbf{M}^{-1} \mathbf{K}_{\mathbf{1}} \mathbf{q}+\mathbf{M}^{-1} \mathbf{K}_{\mathbf{2}}(\mathbf{q}) \mathbf{q}+\mathbf{M}^{-1} \mathbf{K}_{\mathbf{3}}(\mathbf{q}, \mathbf{q}) \mathbf{q} \\
& =\mathbf{M}^{-1} \mathbf{f} \cos (\omega t)
\end{aligned}
$$

The set of nonlinear stiffnesswise coupled (and inertialwise decoupled) equations, given in Eq. (38), is then recast into a new double-dimensional $(2 N)$ set of first-order nonlinear ordinary differential equations via a change of variables through use of $y_{i}=$ $\dot{q}_{i}$ for $i=1,2, \ldots, N$. A high-dimensional reduced-order model is analyzed via choosing $N=32$. More specifically, $2 N=64$ firstorder ordinary differential equations are solved together to obtain the time-varying values of the generalized coordinates. A linear analysis, upon the linear terms of the reduced-order model, is conducted to verify the possibility of the occurrence of internal energy transfer and modal interactions. For the cases tuned to internal resonances, the pseudo-arclength continuation technique is employed to analyze the nonlinear dynamical behavior and internal energy transfer mechanisms of the system via constructing the frequency- and force-response curves. Stability of solution branches is determined via use of the Floquet theory.

The numerical results are obtained for a square epoxy microplate with: $l=17.6 \mu \mathrm{m}$ [48-50], $h=4.0 l, a=300 h, b=300 h$, $E=1.44 \mathrm{GPa}, \rho=1220 \mathrm{~kg} / \mathrm{m}^{3}$, and $\nu=0.38$. Throughout the numerical simulations in this paper, the excitation frequency $\omega$, the forcing amplitude $f_{1}$, the linear natural frequencies for the out-ofplane motion, time $t$, and the damping coefficient $c$ are made dimensionless via

$$
\begin{aligned}
f_{1}^{*} & =\frac{f_{1} b^{4}}{D h}, \quad \Omega=\omega \sqrt{\frac{\rho h b^{4}}{D}}, \quad t^{*}=t \sqrt{\frac{D}{\rho h b^{4}}}, \\
\omega_{i, j}^{*} & =\omega_{i, j} \sqrt{\frac{\rho h b^{4}}{D}}, \quad c^{*}=\frac{c b^{4}}{D} \sqrt{\frac{D}{\rho h b^{4}}}
\end{aligned}
$$

where $D=E h^{3} / 12\left(1-\nu^{2}\right)$. Moreover, the substitution $c^{*}$ $=2 \zeta \omega_{1,1}^{*}$ is made in the numerical simulations. In what follows, the asterisk notations of the dimensionless parameters are dropped for brevity.

For the nonlinear analysis, the following 32 generalized coordinates are used: $u_{2,1}, u_{4,1}, u_{6,1}, u_{8,1}, u_{2,3}, u_{4,3}, u_{2,5}, v_{1,2}, v_{1,4}, v_{1,6}$ $v_{1,8}, v_{3,2}, v_{3,4}, v_{5,2}, w_{1,1}, w_{3,1}, w_{5,1}, w_{1,3}, w_{3,3}, w_{1,5}, \phi_{1_{1,1}}, \phi_{1_{3,1}}$, $\phi_{1_{51}}, \phi_{1_{13}}, \phi_{1_{33}}, \phi_{1_{15}}, \phi_{2_{1,1}}, \phi_{23,}, \phi_{25,1}, \phi_{2_{13}}, \phi_{23,3}$, and $\phi_{2_{15}} ; 64$ first-order nonlinear ordinary differential equations with coupled terms are solved together.

\section{Possibility of the Occurrence of Internal Energy Transfer and Modal Interactions}

The possibility of the occurrence of internal energy transfer and modal interactions is verified by plotting the variations of different natural frequencies for the out-of-plane motion (dimensionless) of the linear system versus the amplitude of the initial curvature; this question is answered that which value of the initial curvature causes internal energy transfer and modal interactions.

Figure $2(a)$ shows that the first possible internal resonance occurs for $A_{0} / h \approx 0.36$, where $\omega_{3,3} \approx 8 \omega_{1,1}$. Figure $2(b)$ illustrates two other possible cases with internal resonances; in particular, an internal resonance in the form of $\omega_{3,1} \approx 4 \omega_{1,1}$ occurs in the 
(a)

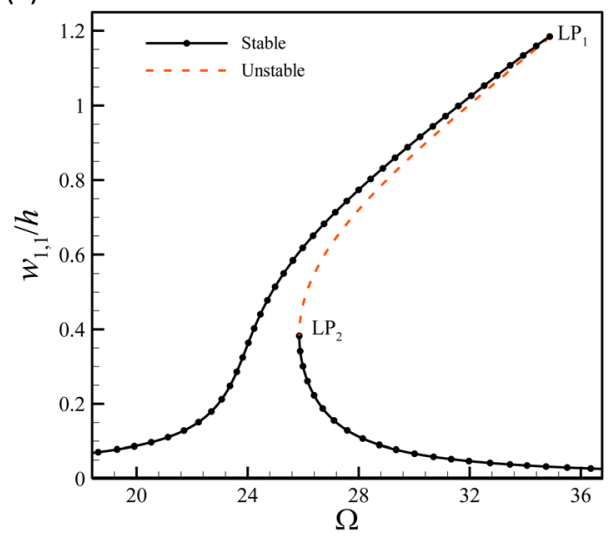

(c)

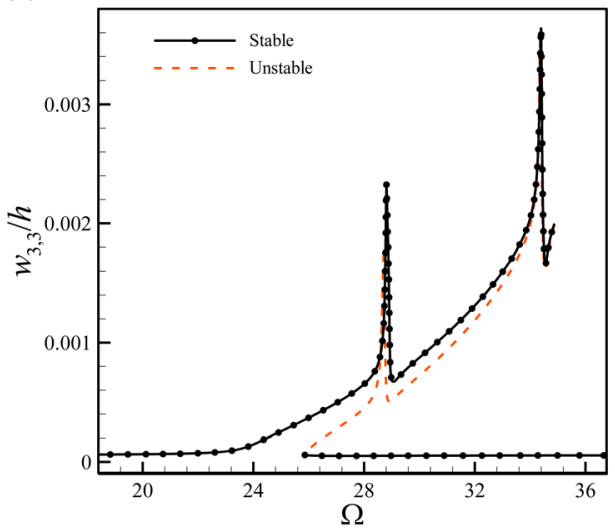

(b)

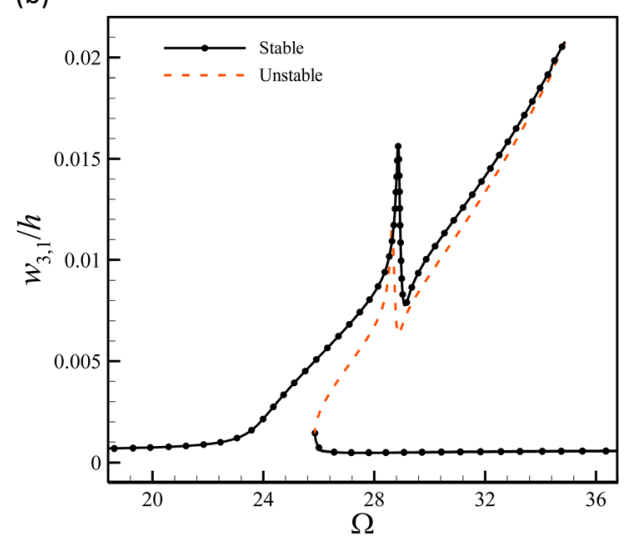

(d)

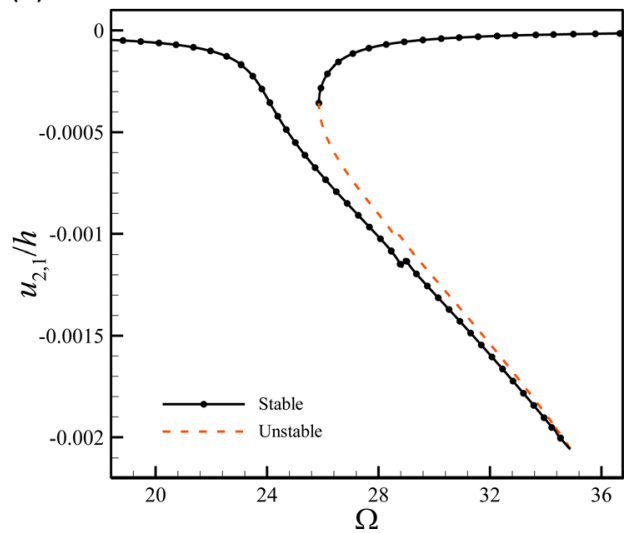

Fig. 3 Frequency-response curves of the system for the generalized coordinates $w_{1,1}, w_{3,1}$, $w_{3,3}$, and $u_{2,1} ; f_{1}=12.00, A_{0} / h=0.36$, and $\omega_{1,1}=24.8747$

vicinity of $A_{0} / h=0.535$. As the amplitude of the initial curvature is increased even further, another possible internal energy transfer in the form of $\omega_{3,1} \approx 3 \omega_{1,1}$ is detected in the vicinity of $A_{0} / h=1.00$. In what follows, the nonlinear dynamical behavior of the system for each of these cases is examined to study the nonlinear effects on such internal energy transfer mechanisms.

\section{Nonlinear Energy Transfer for the Case With $\omega_{3,3} \approx 8 \omega_{1,1}$}

In this section, the frequency- and force-response curves of the system are obtained to illustrate the nonlinear energy transfer between the transverse modes of the system for the case when $A_{0} / h=0.36$ (resulting in $\omega_{3,3} \approx 8 \omega_{1,1}$ ).

The frequency-response curves of the system, when $A_{0} / h=0.36$ and $f_{1}=12.00$, are depicted in Fig. 3, for the generalized coordinates $w_{1,1}, w_{3,1}, w_{3,3}$, and $u_{2,1}$; it should be noted that due to symmetry, $w_{3,1}=w_{1,3}$ and $u_{2,1}=v_{1,2}$. The modal damping ratio of $\zeta=0.008$ is used throughout the numerical simulations. The fundamental natural frequency, i.e., $\omega_{1,1}$, for this case is obtained as 24.8747. It is seen that due to the presence of a nonlinear energy transfer mechanism, extra peaks are observed in the responses of the generalized coordinates $w_{3,1}$ and $w_{3,3}$. In particular, as seen in subfigures $(b)$ and $(c)$, as the frequency of excitation is increased, an internal energy transfer occurs in the vicinity of $\Omega=28.9$, causing an extra peak in the frequency-response curves of $w_{3,1}$ and $w_{3,3}$. By increasing the excitation frequency even further, another internal resonance takes place at $\Omega=34.4$, causing a peak in the response of $w_{3,3}$. Moreover, the system displays a hardening-type nonlinear behavior with two limit point bifurcations at $\Omega=34.8830\left(\mathrm{LP}_{1}\right)$ and $\Omega=25.8604\left(\mathrm{LP}_{2}\right)$-the system response is unstable between the two limit point bifurcations and stable elsewhere.
A comparison between the frequency-response curves of the system obtained via the modified couple stress and classical continuum theories is made by plotting the corresponding curves in Fig. $4 ; \omega_{1,1}=24.8747$ for the modified couple stress theory and $\omega_{1,1}=22.9816$ for the classical continuum theory. It is seen that the classical continuum theory predicts the first internal resonance around $\Omega=26.1$, compared to $\Omega=28.9$ predicted by the modified couple stress theory. Moreover, the second internal resonance occurs in the vicinity of $\Omega=31.2$ when the system is modeled via the classical continuum theory, as opposed to $\Omega=34.4$ for the case of modified couple stress theory. This shows the significance of taking into account the length-scale parameter in order to examine the nonlinear resonant response of a microplate.

Shown in Fig. 5 are the force-response curves of the system for the case with $A_{0} / h=0.36$ and $\Omega=29.1033$. For the ranges of $f_{1}$ studied here, two limit point bifurcations occur at $f_{1}=72.2694$ and 7.1435. Due to the presence of internal energy transfers between transverse modes, extra peaks are observed in the response of $w_{3,1}$ and $w_{3,3}$. In particular, for the $w_{3,1}$ motion (as seen in subfigure $(b)$ ), an energy transfer occurs in the vicinity of $f_{1}=39$, creating a local maximum amplitude in the $w_{3,1}$ motion. For the case of $w_{3,3}$ motion, there are two energy transfer mechanisms around $f_{1}=39$ and $f_{1}=110$, due to the presence of an internal resonance between $\omega_{3,3}$ and $\omega_{1,1}$.

\section{Nonlinear Energy Transfer for the Case With $\omega_{3,1} \approx 4 \omega_{1,1}$}

This section investigates the nonlinear internal energy transfers in the resonant response of the system for the case when the amplitude of the initial curvature is set to $A_{0} / h=0.535$ causing a four-to-one internal resonance in the form of $\omega_{3,1} \approx 4 \omega_{1,1}$. For this case, $\omega_{1,1}=28.0375$. 
(a)

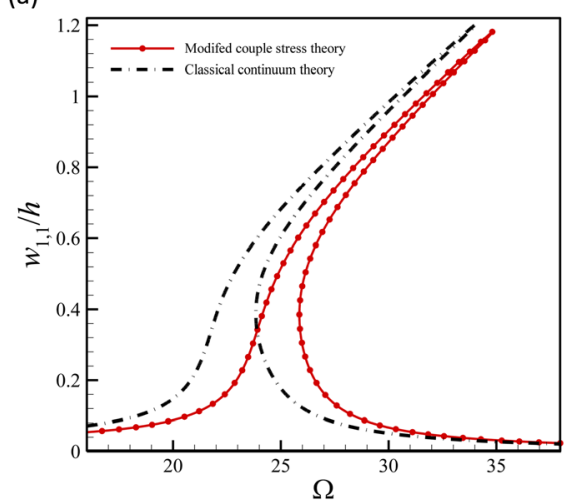

(c)

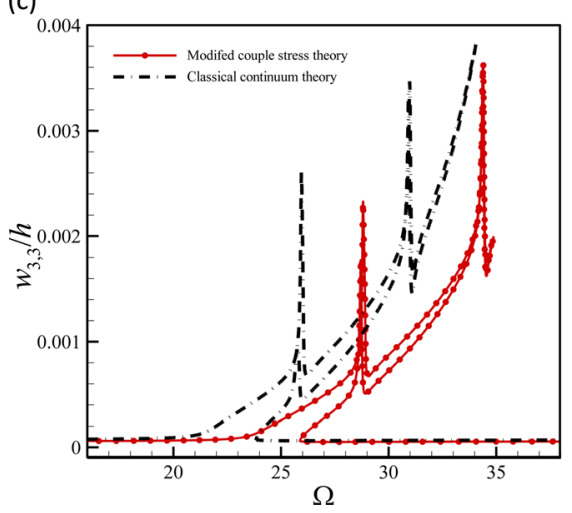

(b)

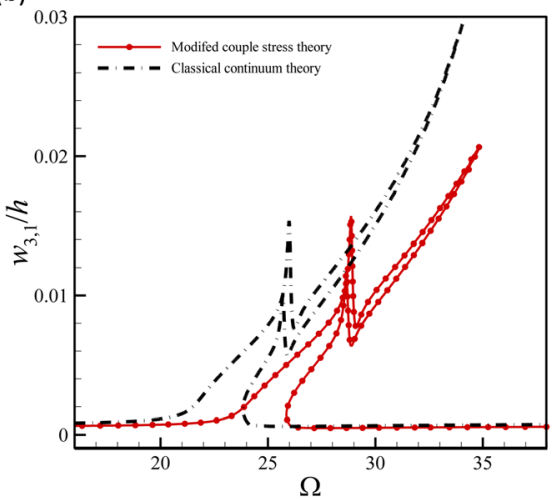

(d)

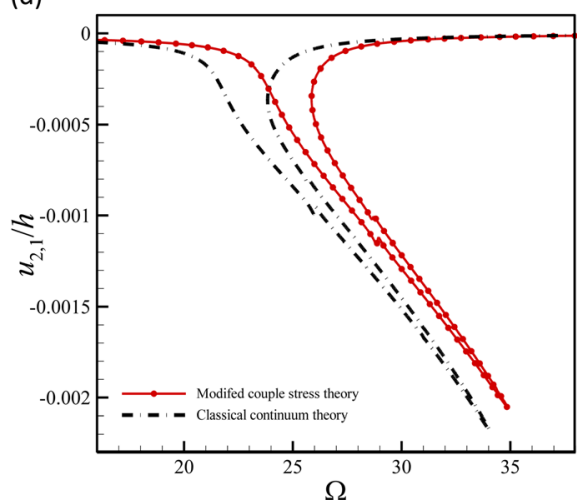

Fig. 4 Frequency-response curves of the system for the generalized coordinates $w_{1,1}$, $w_{3,1}, w_{3,3}$, and $u_{2,1}$, obtained via modified couple stress and classical continuum theories; $f_{1}=12.00$ and $A_{0} / h=0.36$
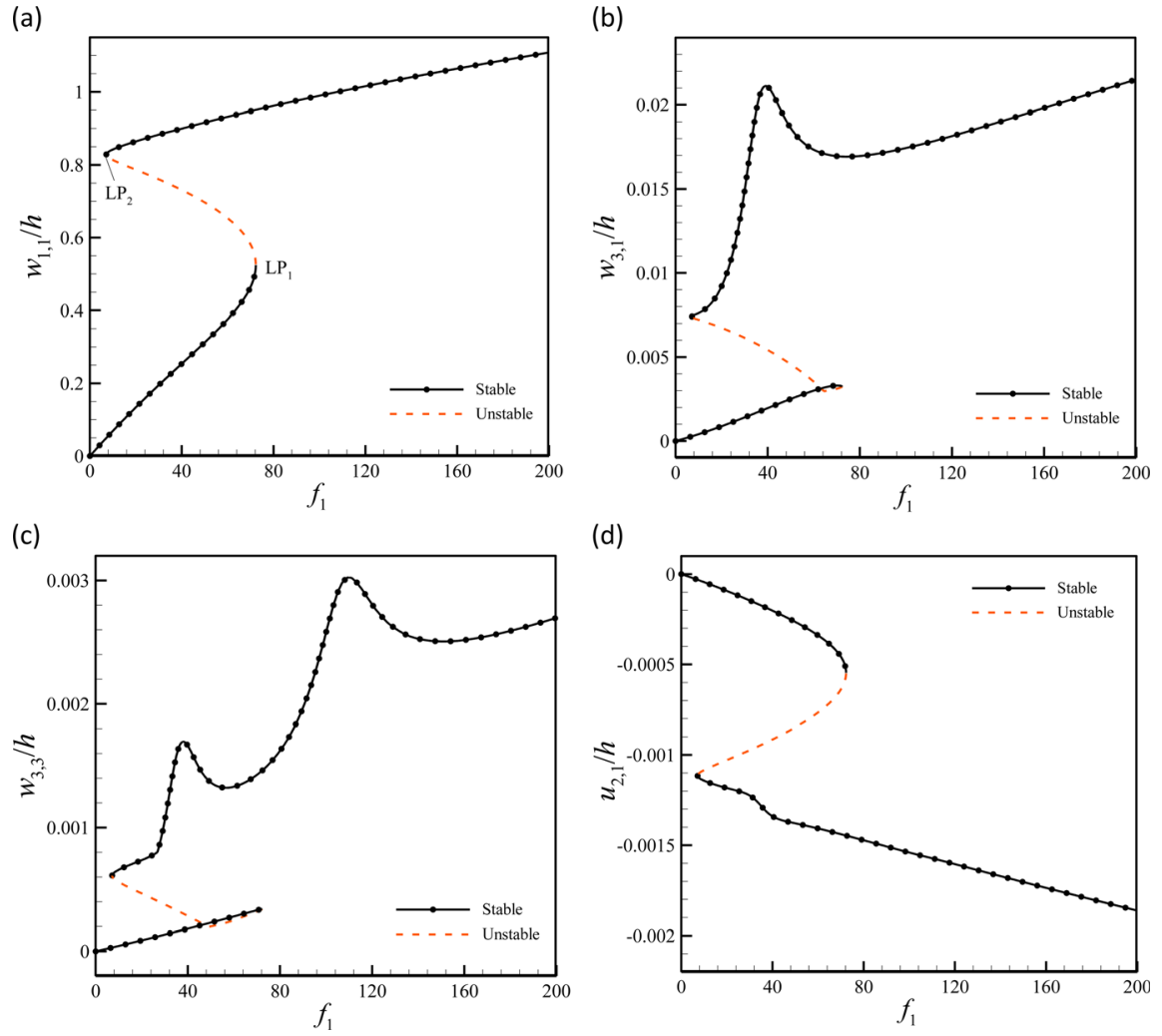

Fig. 5 Force-response curves of the system for the generalized coordinates $w_{1,1}, w_{3,1}$, $W_{3,3}$, and $u_{2,1} ; \Omega=29.1033$ and $A_{0} / h=0.36$ 
(a)

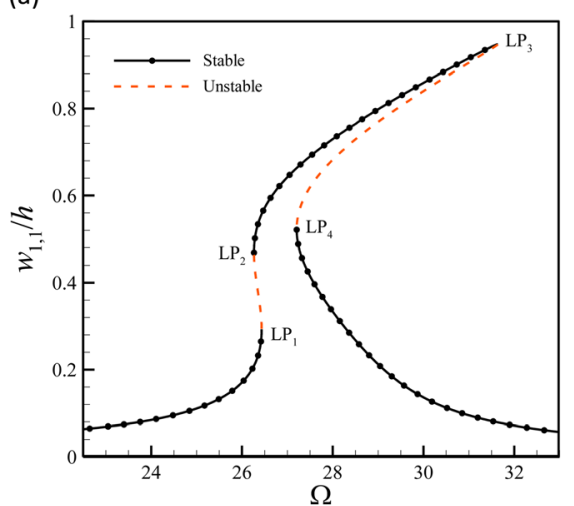

(c)

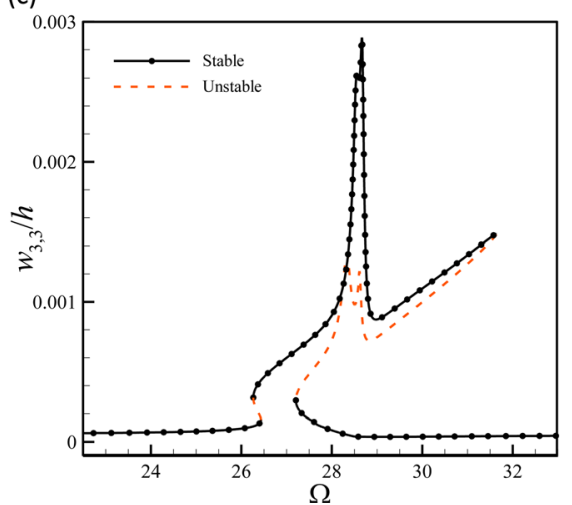

(b)

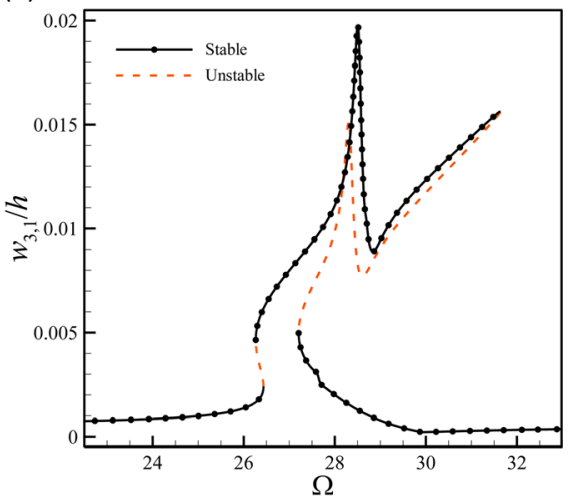

(d)

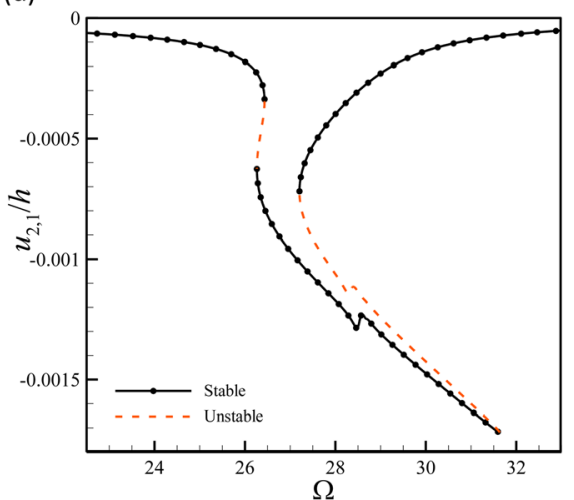

Fig. 6 Frequency-response curves of the system for the generalized coordinates $w_{1,1}$, $w_{3,1}, w_{3,3}$, and $u_{2,1} ; f_{1}=11.00, A_{0} / h=0.535$, and $\omega_{1,1}=28.0375$

(a)

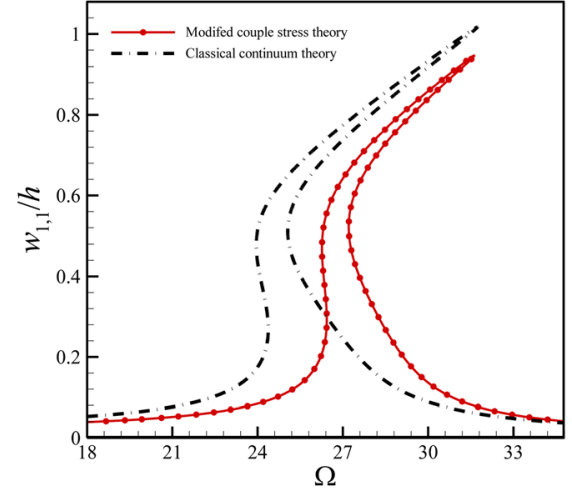

(c)

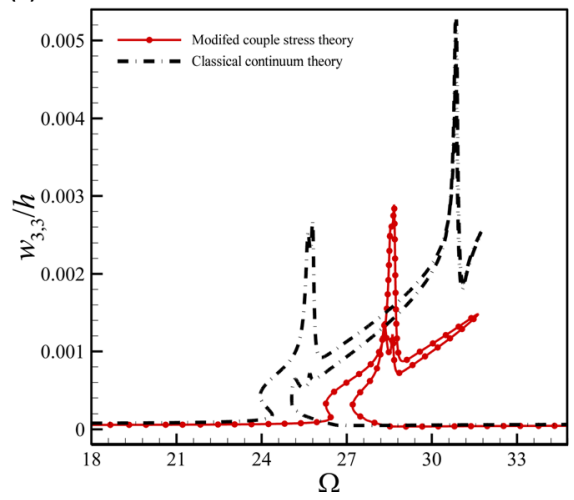

(b)

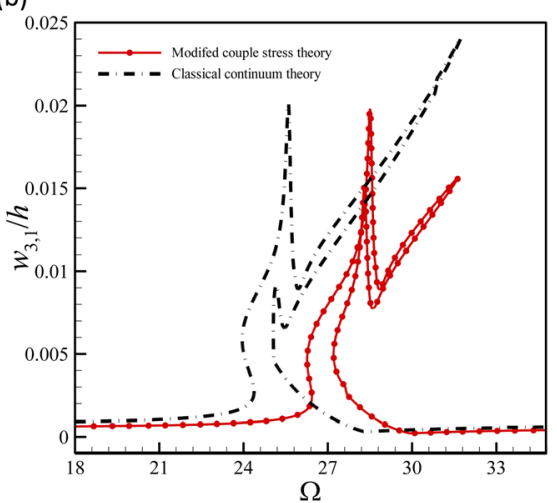

(d)

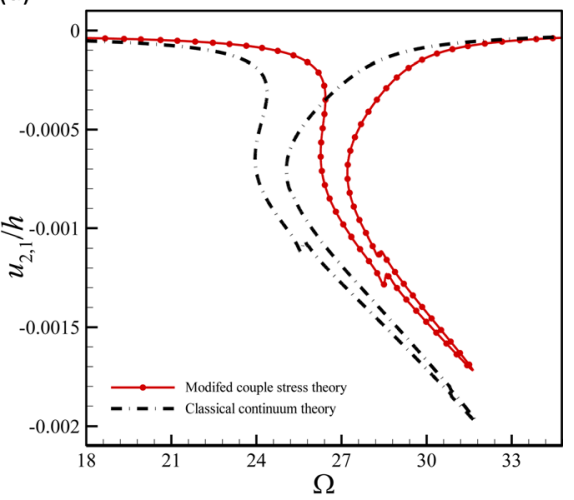

Fig. 7 Frequency-response curves of the system for the generalized coordinates $w_{1,1}$, $w_{3,1}, w_{3,3}$, and $u_{2,1}$, obtained via modified couple stress and classical continuum theories; $f_{1}=11.00$ and $A_{0} / h=0.535$ 
(a)

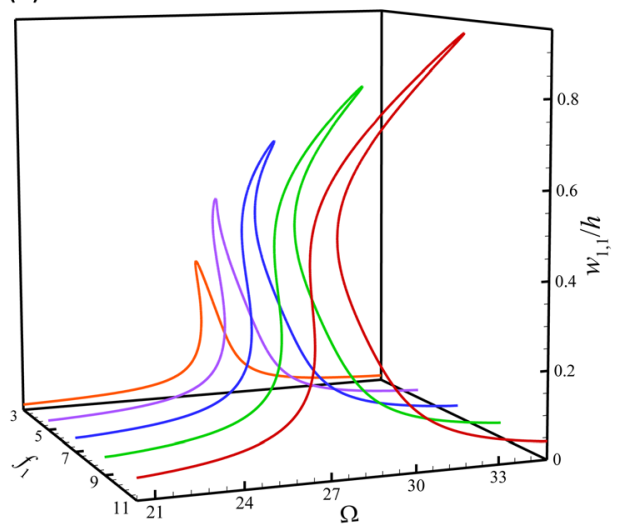

(c)

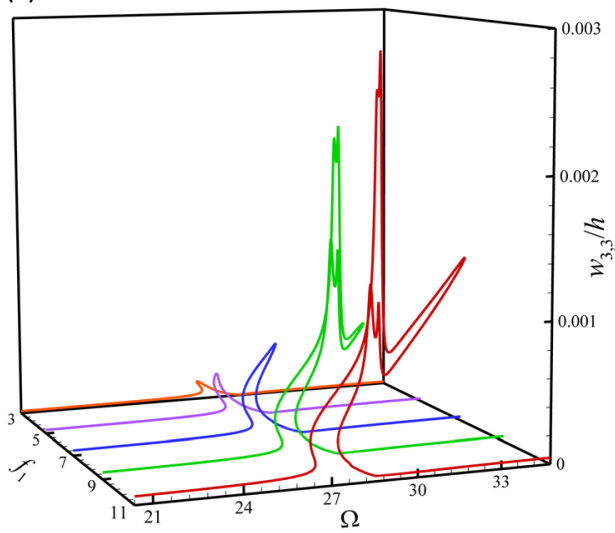

(b)

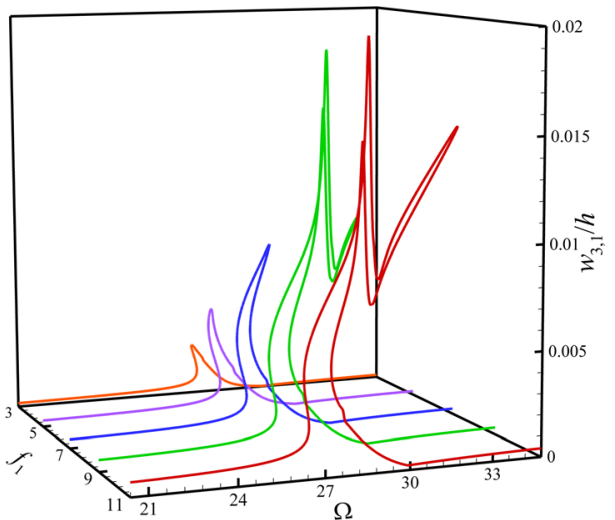

(d)

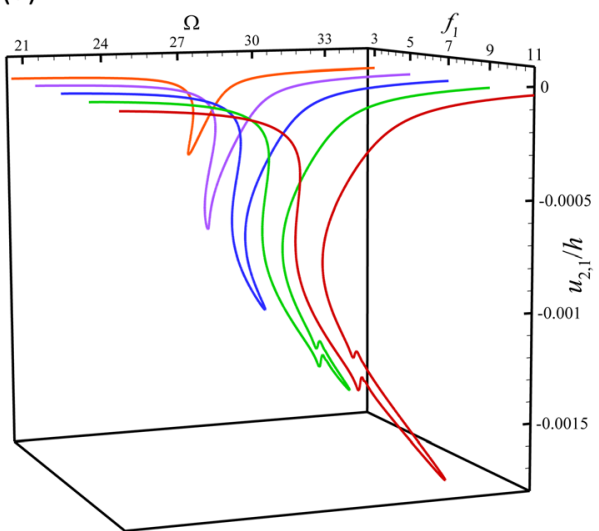

Fig. 8 The effect of forcing amplitude on the frequency-response curves of the system for the generalized coordinates $w_{1,1}, w_{3,1}, w_{3,3}$, and $u_{2,1} ; A_{0} / h=0.535$ and $\omega_{1,1}=28.0375$

Figure 6 shows the frequency-response curves of the system where $A_{0} / h=0.535$ and $f_{1}=11.00$. Compared to the system of Fig. 3, it is seen that due to increased amplitude of the initial curvature, the system displays an initial softening behavior followed by a hardening one. Moreover, four limit point bifurcations occur as a result of varying the excitation frequency, at $\Omega=26.4336$ $\left(\mathrm{LP}_{1}\right), \Omega=26.2643\left(\mathrm{LP}_{2}\right), \Omega=31.6375\left(\mathrm{LP}_{3}\right)$, and $\Omega=27.2057$ $\left(\mathrm{LP}_{4}\right)$. It is seen in subfigures $(b)$ and $(c)$ that there is an extra peak in the response of $w_{3,1}$ and $w_{3,3}$ generalized coordinates around $\Omega=28.5$ due to presence of an internal resonance between $\omega_{3,1}$ and $\omega_{1,1}$. It is interesting to note that for both $w_{3,1}$ and $w_{3,3}$ motions, the maximum amplitude due to occurrence of the internal resonance is more than that due to the nonlinear resonance (i.e., $\mathrm{LP}_{3}$ ), showing the importance of internal energy transfers in the resonant response of microplates. Figure 7 shows a comparison between the results obtained via the modified couple stress and classical continuum theories. It is seen that the frequencyresponse curves are shifted to the left, since the natural frequency predicted by the classical continuum theory is smaller than that predicted by the modified couple stress theory (which takes into account small size effects). Hence, as seen in subfigure $(b)$, the classical continuum theory predicts the internal energy transfer at a smaller excitation frequency, compared to the modified couple stress theory. Moreover, as seen in subfigure $(c)$, two energy transfer mechanisms are detected by the classical continuum theory, while the modified couple stress theory predicts only one internal energy transfer mechanism.

Figure 8 illustrates the frequency-response of the system for several forcing amplitudes. It is seen that for small values of $f_{1}$, the system displays only a softening-type nonlinear behavior. As the forcing amplitude is set to larger values, the system displays a secondary hardening-type behavior as well as an internal energy transfer. It is worthwhile noting that the internal resonance has been detected only when the forcing amplitude is set to larger values.

\section{Nonlinear Energy Transfer for the Case With $\omega_{3,1} \approx 3 \omega_{1,1}$}

This section examines the nonlinear energy transfer mechanisms between the transverse modes when there is a three-to-one internal resonance in the form of $\omega_{3,1} \approx 3 \omega_{1,1}$, due to presence of an initial curvature with the amplitude of $A_{0} / h=1.00$.

Figure 9 shows the frequency-response plots of the system with $A_{0} / h=1.00, f_{1}=14.50$, and $\omega_{1,1}=39.3379$. As seen in this figure, the system displays a softening-type nonlinear behavior, due to increased amplitude of the initial curvature, with two limit point bifurcations at $\Omega=37.3226\left(\mathrm{LP}_{1}\right)$ and $\Omega=28.6435\left(\mathrm{LP}_{2}\right)$. As shown in the figure, an internal energy transfer occurs in the vicinity of $\Omega=37$, causing extra peaks in the frequency-response of the $w_{1,1}$ and $w_{3,1}$ motions. There is also another energy transfer mechanism around $\Omega=32.7$, which only affects the $w_{3,1}$ generalized coordinate.

\section{Validations}

The numerical simulations employed in this study are validated by comparing the frequency-response of the $w_{1,1}$ motion of a macroplate (hence way simpler model) obtained by the present study and plotted versus that given in Ref. [51] in Fig. 10. The figure shows that the results match perfectly, hence proving the validity of the numerical simulations employed in the present study.

\section{Conclusions}

Internal energy transfer and modal interactions in the dynamical behavior of slightly curved shear deformable microplates have 
(a)

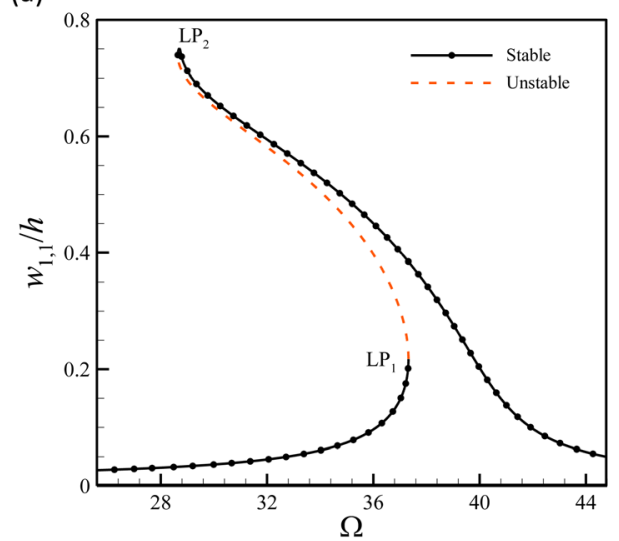

(c)

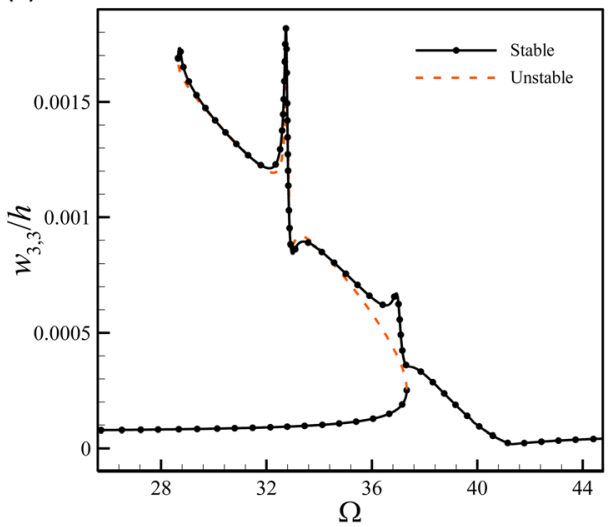

(b)

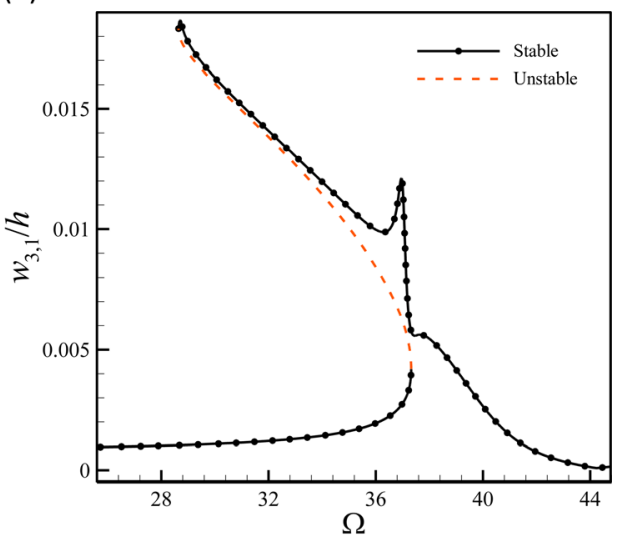

(d)

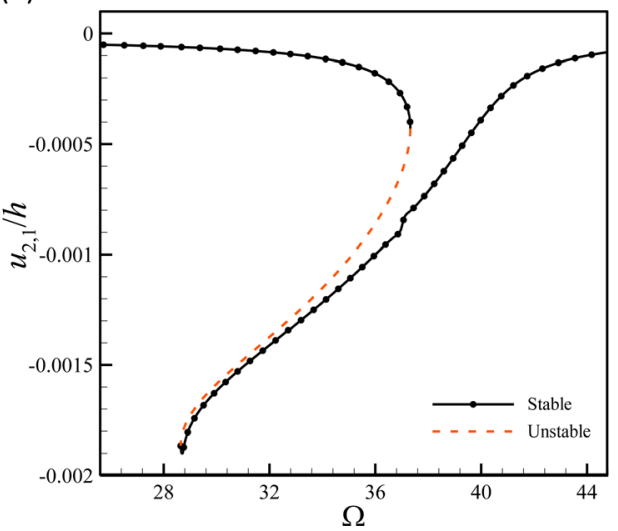

Fig. 9 Frequency-response curves of the system for the generalized coordinates $w_{1,1}, w_{3,1}$, $w_{3,3}$, and $u_{2,1} ; f_{1}=14.50, A_{0} / h=1.00$, and $\omega_{1,1}=39.3379$

been investigated numerically in this paper. On the basis of the modified couple stress theory, the third-order shear deformation theory was employed to derive the continuous and reduced-order models of the system by retaining the in-plane displacements and inertia. The initial curvature was modeled by an initial displacement in the out-of-plane direction. A model reduction was conducted via Lagrange's equations along with an assumed-mode technique. The reduced-order model was then solved numerically through use of the pseudo-arclength continuation. Stability analysis was conducted via the Floquet theory. The possibility of the occurrence of modal interactions and internal energy transfers was

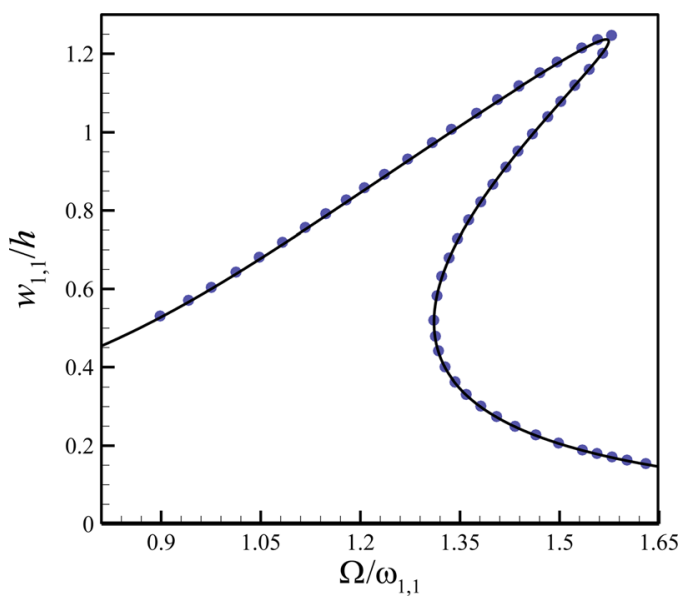

Fig. 10 Frequency-response of a macroplate for the $w_{1,1}$ mode: solid line and symbols represent the results obtained by the numerical simulations employed in the present study and those given in Ref. [51], respectively verified by means of a linear analysis via obtaining different natural frequencies of the system and determining their ratio as a function of the initial curvature. The nonlinear resonant response of the system was obtained for different cases with internal energy transfer, showing that: (i) depending on the amplitude of the initial curvature, the system displays softening, hardening, or softeninghardening behaviors; (ii) due to the presence of internal energy transfers, extra peaks appear in the frequency- and forceresponses; (iii) the maximum response amplitude due to an internal energy transfer is more than that due to a nonlinear resonance for some cases; (iv) the modified couple stress theory predicts the occurrence of the internal energy transfer at larger frequencies, compared to the classical continuum theory; (v) the energy transfer due to internal resonances is detected only at sufficiently large forcing amplitudes.

\section{Acknowledgment}

The financial support to this research by the start-up grant of the University of Wollongong is gratefully acknowledged.

\section{References}

[1] Rembe, C., and Muller, R. S., 2002, "Measurement System for Full ThreeDimensional Motion Characterization of MEMS," J. Microelectromech. Syst., 11(5), pp. 479-488.

[2] Ghayesh, M. H., Farokhi, H., and Amabili, M., 2013, "Nonlinear Behaviour of Electrically Actuated MEMS Resonators," Int. J. Eng. Sci., 71, pp. 137-155.

[3] Younis, M. I., Abdel-Rahman, E. M., and Nayfeh, A., 2003, "A Reduced-Order Model for Electrically Actuated Microbeam-Based MEMS," J. Microelectromech. Syst., 12(5), pp. 672-680.

[4] De, S. K., and Aluru, N. R., 2004, "Full-Lagrangian Schemes for Dynamic Analysis of Electrostatic MEMS," J. Microelectromech. Syst., 13(5), pp. 737-758.

[5] Liu, S., Davidson, A., and Lin, Q., 2004, "Simulation Studies on Nonlinear Dynamics and Chaos in a MEMS Cantilever Control System," J. Micromech. Microeng., 14(7), pp. 1064-1073. 
[6] Sulfridge, M., Saif, T., Miller, N., and Meinhart, M., 2004, "Nonlinear Dynamic Study of a Bistable MEMS: Model and Experiment," J. Microelectromech. Syst., 13(5), pp. 725-731.

[7] Zhang, W. M., and Meng, G., 2007, "Nonlinear Dynamic Analysis of Electrostatically Actuated Resonant MEMS Sensors Under Parametric Excitation," IEEE Sens. J., 7(3), pp. 370-380.

[8] Duwel, A., Candler, R. N., Kenny, T. W., and Varghese, M., 2006, "Engineering MEMS Resonators With Low Thermoelastic Damping," J. Microelectromech. Syst., 15(6), pp. 1437-1445.

[9] Mestrom, R. M. C., Fey, R. H. B., van Beek, J. T. M., Phan, K. L., and Nijmeijer, H., 2008, "Modelling the Dynamics of a MEMS Resonator: Simulations and Experiments," Sens. Actuators, A, 142(1), pp. 306-315.

[10] Bergers, L. I. J. C., Hoefnagels, J. P. M., Delhey, N. K. R., and Geers, M. G. D., 2011, "Measuring Time-Dependent Deformations in Metallic MEMS," Microelectron. Reliab., 51(6), pp. 1054-1059.

[11] Haghighi, H. S, and Markazi, A. H. D., 2010 "Chaos Prediction and Control in MEMS Resonators," Commun. Nonlinear Sci. Numer. Simul., 15(10), pp. 3091-3099.

[12] Farokhi, H., and Ghayesh, M., "Size-Dependent Behaviour of Electrically Actuated Microcantilever-Based MEMS," Int. J. Mech. Mater. Des. (in press)

[13] Gholipour, A., Farokhi, H., and Ghayesh, M., 2014, "In-Plane and Out-OfPlane Nonlinear Size-Dependent Dynamics of Microplates," Nonlinear Dyn. 79(3), pp. 1771-1785.

[14] Ghayesh, M. H., and Farokhi, H., 2015, "Nonlinear Dynamics of Microplates," Int. J. Eng. Sci., 86, pp. 60-73.

[15] Farokhi, H., and Ghayesh, M. H., 2015, "Nonlinear Dynamical Behaviour of Geometrically Imperfect Microplates Based on Modified Couple Stress Theory," Int. J. Mech. Sci., 90, pp. 133-144.

[16] Ghayesh, M. H., Farokhi, H., and Alici, G., 2015, "Subcritical Parametric Dynamics of Microbeams," Int. J. Eng. Sci., 95, pp. 36-48.

[17] Das, K., and Batra, R. C., 2009, "Pull-in and Snap-Through Instabilities in Transient Deformations of Microelectromechanical Systems," J. Micromech. Microeng., 19(3), p. 035008.

[18] Slava, K., Bojan, R. I., David, S., Shimon, S., and Harold, C., 2008, "The PullIn Behavior of Electrostatically Actuated Bistable Microstructures," J. Micromech. Microeng., 18(5), p. 055026.

[19] Lam, D. C. C., Yang, F., Chong, A. C. M., Wang, J., and Tong, P., 2003, "Experiments and Theory in Strain Gradient Elasticity," J. Mech. Phys. Solids, 51(8), pp. 1477-1508.

[20] McFarland, A. W., and Colton, J. S., 2005, "Role of Material Microstructure in Plate Stiffness With Relevance to Microcantilever Sensors," J. Micromech. Microeng., 15(5), p. 1060.

[21] Fleck, N. A., Muller, G. M., Ashby, M. F., and Hutchinson, J. W., 1994, "Strain Gradient Plasticity: Theory and Experiment," Acta Metall. Mater., 42(2), pp. 475-487.

[22] Zhao, J., Zhou, S., Wang, B., and Wang, X., 2012, "Nonlinear Microbeam Model Based on Strain Gradient Theory," Appl. Math. Modell., 36(6), pp. 2674-2686.

[23] Akgöz, B., and Civalek, Ö., 2013, "A Size-Dependent Shear Deformation Beam Model Based on the Strain Gradient Elasticity Theory," Int. J. Eng. Sci., 70, pp. 1-14.

[24] Salamat-Talab, M., Shahabi, F., and Assadi, A., 2012, "Size Dependent Analysis of Functionally Graded Microbeams Using Strain Gradient Elasticity Incorporated With Surface Energy," Appl. Math. Modell., 37(1-2), pp. 507-526.

[25] Mohammadi, H., and Mahzoon, M., 2013, "Thermal Effects on Postbuckling of Nonlinear Microbeams Based on the Modified Strain Gradient Theory," Compos. Struct., 106, pp. 764-776.

[26] Farokhi, H., Ghayesh, M. H., and Amabili, M., 2013, "Nonlinear Dynamics of Geometrically Imperfect Microbeam Based on the Modified Couple Stress Theory," Int. J. Eng. Sci., 68, pp. 11-23.

[27] Ghayesh, M. H., Farokhi, H., and Amabili, M., 2014, "In-Plane and Out-ofPlane Motion Characteristics of Microbeams With Modal Interactions," Compos. Part B: Eng., 60, pp. 423-439.

[28] Ghayesh, M. H., Amabili, M., and Farokhi, H., 2013, "Three-Dimensional Nonlinear Size-Dependent Behaviour of Timoshenko Microbeams,” Int. J. Eng. Sci., 71, pp. 1-14.
[29] Hashemi, S. H., and Samaei, A. T., 2011, "Buckling Analysis of Micro/Nanoscale Plates Via Nonlocal Elasticity Theory," Physica E, 43(7), pp. 1400-1404.

[30] Sharma, J. N., and Sharma, R., 2011, "Damping in Micro-Scale Generalized Thermoelastic Circular Plate Resonators," Ultrasonics, 51(3), pp. 352-358.

[31] He, L., Lou, J., Zhang, E., Wang, Y., and Bai, Y., 2015, “A Size-Dependent Four Variable Refined Plate Model for Functionally Graded Microplates Based on Modified Couple Stress Theory," Compos. Struct., 130, pp. 107-115.

[32] Wang, B., Zhou, S., Zhao, J., and Chen, X., 2011, "A Size-Dependent Kirchhoff Micro-Plate Model Based on Strain Gradient Elasticity Theory," Eur. J. Mech. A. Solids, 30(4), pp. 517-524

[33] Ramezani, S., 2012, "A Shear Deformation Micro-Plate Model Based on the Most General Form of Strain Gradient Elasticity," Int. J. Mech. Sci., 57(1) pp. 34-42.

[34] Tahani, M., Askari, A. R., Mohandes, Y., and Hassani, B., 2015, "SizeDependent Free Vibration Analysis of Electrostatically Pre-Deformed Rectangular Micro-Plates Based on the Modified Couple Stress Theory," Int. J. Mech. Sci., 94-95, pp. 185-198.

[35] Roque, C. M. C., Ferreira, A. J. M., and Reddy, J. N., 2013, "Analysis of Mindlin Micro Plates With a Modified Couple Stress Theory and a Meshless Method," Appl. Math. Modell., 37(7), pp. 4626-4633.

[36] Asghari, M., 2012, "Geometrically Nonlinear Micro-Plate Formulation Based on the Modified Couple Stress Theory,” Int. J. Eng. Sci., 51, pp. 292-309.

[37] Thai, H.-T., and Choi, D.-H., 2013, "Size-Dependent Functionally Graded Kirchhoff and Mindlin Plate Models Based on a Modified Couple Stress Theory," Compos. Struct., 95, pp. 142-153.

[38] Ansari, R., Gholami, R., Faghih Shojaei, M., Mohammadi, V., and Darabi, M. A., 2015, "Size-Dependent Nonlinear Bending and Postbuckling of Functionally Graded Mindlin Rectangular Microplates Considering the Physical Neutral Plane Position," Compos. Struct., 127, pp. 87-98.

[39] Das, K., and Batra, R. C., 2009, "Symmetry Breaking, Snap-Through and PullIn Instabilities Under Dynamic Loading of Microelectromechanical Shallow Arches," Smart Mater. Struct., 18(11), p. 115008.

[40] Ouakad, H. M., and Younis, M. I., 2010, "The Dynamic Behavior of MEMS Arch Resonators Actuated Electrically,” Int. J. Non Linear Mech., 45(7), pp. 704-713.

[41] Ouakad, H. M., and Younis, M. I., 2014, "On Using the Dynamic SnapThrough Motion of MEMS Initially Curved Microbeams for Filtering Applications," J. Sound Vib., 333(2), pp. 555-568.

[42] Ouakad, H. M., 2013, "An Electrostatically Actuated MEMS Arch Band-Pass Filter,” Shock Vib., 20(4), pp. 809-819.

[43] Mohammad, T., and Ouakad, H., 2014, "Static, Eigenvalue Problem and Bifurcation Analysis of MEMS Arches Actuated by Electrostatic Fringing-Fields," Microsyst. Technol.

[44] Medina, L., Gilat, R., Ilic, B., and Krylov, S., 2014, "Experimental Investigation of the Snap-Through Buckling of Electrostatically Actuated Initially Curved Pre-Stressed Micro Beams," Sens. Actuators, A, 220, pp. 323-332.

[45] Medina, L., Gilat, R., and Krylov, S., 2014, "Symmetry Breaking in an Initially Curved Pre-Stressed Micro Beam Loaded by a Distributed Electrostatic Force," Int. J. Solids Struct., 51(11-12), pp. 2047-2061.

[46] Medina, L., Gilat, R., and Krylov, S., 2012, "Symmetry Breaking in an Initially Curved Micro Beam Loaded by a Distributed Electrostatic Force," Int. J. Solid Struct., 49(13), pp. 1864-1876.

[47] Yang, F., Chong, A. C. M., Lam, D. C. C., and Tong, P., 2002, "Couple Stress Based Strain Gradient Theory for Elasticity," Int. J. Solids Struct., 39(10), pp. 2731-2743.

[48] Park, S. K., and Gao, X. L., 2006, "Bernoulli-Euler Beam Model Based on a Modified Couple Stress Theory," J. Micromech. Microeng., 16(11), p. 2355.

[49] Ma, H. M., Gao, X. L., and Reddy, J. N., 2008, "A Microstructure-Dependent Timoshenko Beam Model Based on a Modified Couple Stress Theory," J. Mech. Phys. Solids, 56(12), pp. 3379-3391.

[50] Gao, X. L., Huang, J. X., and Reddy, J. N., 2013, "A Non-Classical ThirdOrder Shear Deformation Plate Model Based on a Modified Couple Stress Theory," Acta Mech., 224(11), pp. 2699-2718.

[51] Amabili, M., 2004, "Nonlinear Vibrations of Rectangular Plates With Different Boundary Conditions: Theory and Experiments," Comput. Struct., 82(31-32), pp. 2587-2605. 